\title{
Laboratory Test Reference Range Upper Limit Standard Unit
}

National Cancer Institute

\section{Source}

National Cancer Institute. Laboratory Test Reference Range Upper Limit Standard Unit. NCl Thesaurus. Code C83317.

The greatest value in a range of expected results for a particular laboratory test in standard units. 